\title{
Schwannoma presenting as unilateral nasal blockage: a case report and review of literature
}

\begin{abstract}
Schwannomas are neurogenic tumours which are rarely found in the nose and paranasal sinuses. We would like to share our experience of managing a patient with maxillary sinus schwannoma by describing this case report along relevant summary of the published literature. A 39-year-old female presented with chronic rhinosinusitis affecting her right nose. After failed medical therapy, surgery was planned and a CT scan of her sinuses was requested pre-operatively. The CT scan revealed complete opacification of the right maxillary sinus and a biopsy confirmed it to be a schwannoma. The lesion was removed using a combined endoscopic and Caldwell-Luc approach. The patient made a good post-operative recovery and remained disease free at lyear follow-up. We conclude that Schwannoma of the maxillary sinus remains one of the differential diagnoses for a unilateral opacification of the maxillary sinus. Every effort should be made to diagnose this condition pre-operatively so that patient can be counselled accordingly and appropriate surgical intervention can be planned.
\end{abstract}

Volume 2 Issue I - 2015

\author{
VVallamkondu,' M Shakeel,' D Houghton ${ }^{2}$ \\ 'Department of otolaryngology-head \& neck surgery, Aberdeen \\ Royal Infirmary, UK \\ ${ }^{2}$ Department of otolaryngology-head \& neck surgery, Southern \\ General Hospital, UK
}

\begin{abstract}
Correspondence: Vamsidhar Vallamkondu, Department of otolaryngology-head \& neck surgery,Ward 45, Aberdeen Royal Infirmary, AB25 2ZN, UK, Tel 0044I224552100, Fax
\end{abstract} 0044I224554569, Email vams2I@doctors.org.uk

Received: January 03, 2015 | Published: January 21, 2015

Keywords: maxillary sinus, schwannoma, surgery, caldwell-luc approach

\section{Introduction}

Schwannomas are slow growing benign tumours and originate from the Schwann cells in the sheath of the myelinated nerve fibres. These tumours can occur anywhere in the body but are relatively common $(25-45 \%)$ in the head and neck region but involvement of the sinonasal region is rare $(4 \%))^{1,2}$ Initially the patients experience vague nasal symptoms and the specialist help is sought only once the conservative means fail to improve the symptoms. Sinonasal anatomy makes the early clinical diagnosis of such tumours difficult and by the time a definite diagnosis is made, the sinonasal schwannomas are quite large. ${ }^{1,2}$ We share our experience of managing such a patient who was initially thought to have chronic Rhinosinusitis but the definite underlying pathology was a schwannoma originating from the right maxillary sinus.

\section{Case report}

A 39year old Caucasian female presented with a few years history of progressive right sided nasal blockage with no associated history of epistaxis, headache or pain. She was a non-smoker and otherwise fit and well. On examination, there was decreased air entry on the right side. Nasal endoscopy revealed a straight nasal septum but the right nasal cavity was smaller compared to the left side. Endoscopically, no intranasal lesion was identified but because of the bulge in the right lateral nasal wall, a computed tomogram (CT) of her paranasal sinuses was arranged. The CT scan revealed soft tissue opacity in the right maxillary sinus with expansion of the sinus into the right nasal cavity but no evidence of bony erosion (Figures $1 \& 2$ ).

Examination under general anaesthesia confirmed a bulge on the right lateral wall of nose, pushing the middle and inferior turbinates medially. Uncinectomy was performed followed by middle meatal antrostomy on the right side which revealed a globular mass lesion filling the maxillary antrum (Figure 3). The tumor was firm in consistency but no contact bleeding was noticed. At this stage it was decided to carry out the biopsy only. The lesion was confirmed to be a benign schwannoma on histological examination.

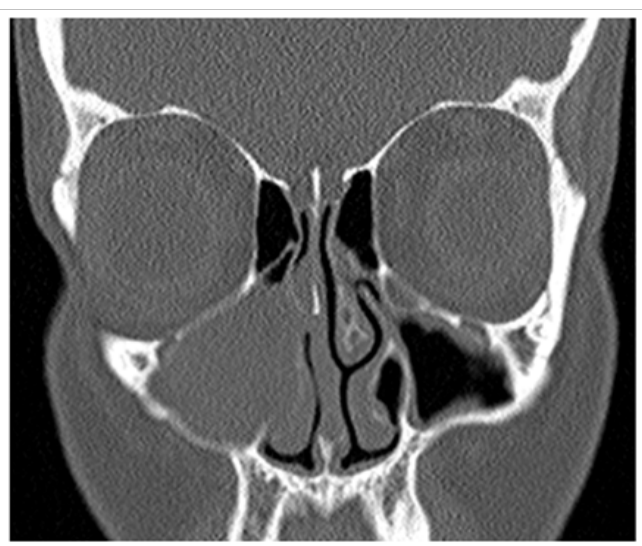

Figure I CT shows maxillary sinus tumour pushing the right lateral nasa wall medially.

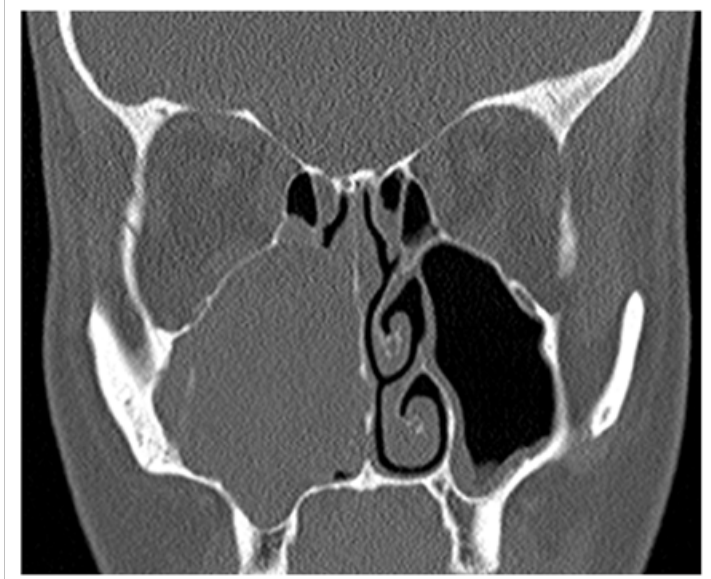

Figure 2 CT scan shows complete opacification of the right maxillary sinus and nasal cavity. 


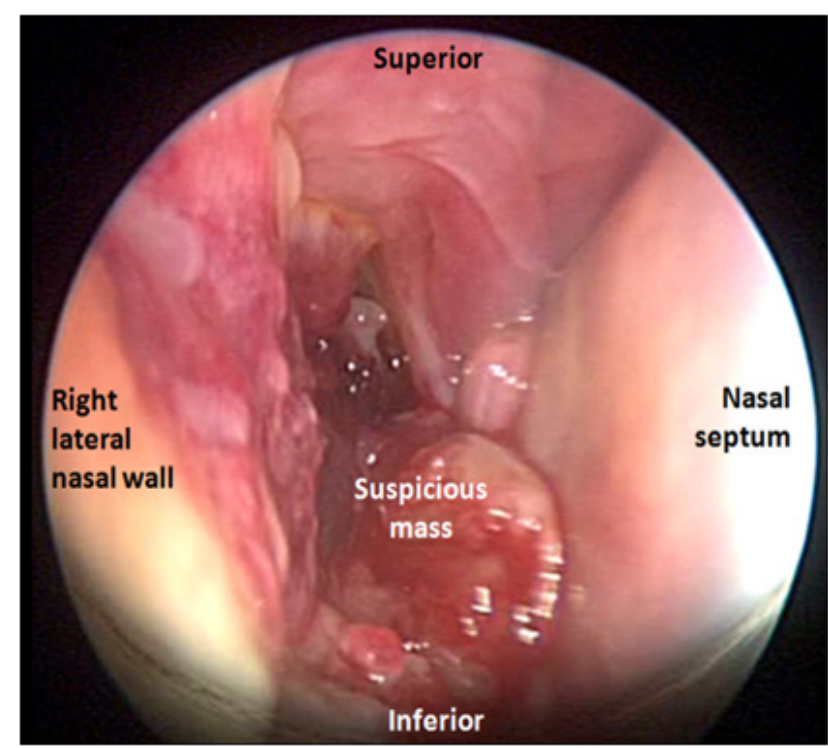

Figure 3 Intraoperative view of the right nasal cavity showing a globular mass.

Combined endoscopic and Caldwell Luc approach was employed to successfully excise the tumour completely. A sublabial incision was used $1 \mathrm{~cm}$ above the gingivolabial groove starting from the level of lateral incisors to the $2^{\text {nd }}$ molar tooth. This prevents the damage to the nerves and postoperative numbness. A window was made over the canine fossa to approach the tumour. With the help of Hopkins rod, the tumour was visualised through middle meatus antrostomy. The tumour was well encapsulated measuring about $45 \mathrm{~mm} \times 35 \mathrm{~mm}$ x $25 \mathrm{~mm}$ size. The mass was mobilised all around. The window through the anterior wall of maxilla was not large enough for the tumour to be removed in total. Therefore, the mass was debulked using the mircodebrider and once small enough the tumour was removed through the anterior wall of maxilla. The nerve of origin was not identifiable. The maxillary sinus and the middle meatus were packed with biodegradable dressing (Nasopore). The sub labial incision was closed with Vicryl. The postoperative period was uneventful. The formal pathology of the specimen confirmed the tumour as benign schwannoma.

The patient made an uneventful recovery and was discharged home on simple analgesia. No post operative complications were reported and the patient returned to her normal functional level using nasal endoscopy, the patient remained under clinical surveillance to monitor any disease recurrence. To the best of our knowledge at 2 years follow up there had not been any schwannoma recurrence and no post operative scans were deemed necessary.

\section{Discussion}

A schwannoma, also known as Neurilemmoma, is a benign tumour arising from the Schwann cells of the nerve sheath. It was first described as a distinct pathological entity by Verocay. Schwannomas of the head and neck are relatively uncommon and are not to be confused with neurofibromas with which they share a common precursor; the Schwann cell. Approximately $40 \%$ of these tumours occur in the head and neck region and only $4 \%$ of head and neck Schwannomas originate from the sinonasal tract, ${ }^{1,2}$ where the ethmoid, maxillary sinus, nasal fossa and sphenoid sinus are involved in decreasing order. ${ }^{3}$ Donnelly et al., ${ }^{4}$ found only 32 cases of Schwannomas occurring in paranasal sinuses. To our knowledge, only six cases of Schwannomas arising solely from maxillary sinus have been reported. ${ }^{5}$
Nasal and paranasal Schwannomas give rise to various signs and symptoms that relate to the anatomic site involved; the nerve of origin or compression of adjacent nerves. The most common symptoms are nasal obstruction, epistaxis, hyposmia and pain. ${ }^{1}$ Epistaxis is usually associated with ethmoid and nasal fossa tumours. The unilateral nature of nasal symptoms is always worrying and persistence of symptoms warrant detailed investigations including endoscopy and radiology. The CT and MRI scans are complementary and help document the nature and extent of the disease. The definite diagnosis requires biopsy of the lesion.

Schwannomas consist of a true capsule facilitating the surgical dissection. They have a hard parenchymatous consistency with a non-infiltrating nature and exhibit a smooth surface under a normal skin. The differential diagnosis include fibroma, chondroma, and leiomyoma. Definitive diagnosis is based on the histological and immunohistochemical findings. It is important to understand that these tumours do not entrap the nerve axons, but rather push them aside. The clinical significance of this is that the nerve may be surgically preserved. The typical schwannoma exhibits a biphasic histologic pattern of Antoni A and Antoni B areas. Antoni A tissue is composed of palisading spindle-shaped Schwann cells surrounding an acellular central region. This complete unit is called a Verocay body. Antoni B tissue is less cellular and forms no distinctive pattern. S-100 protein is a neural-crest marker antigen present in the supporting cells of the nervous system and is an important diagnostic tool, as Schwannomas show intense immuno-staining for S-100. ${ }^{4}$ However, malignant tumours stain less intensely for S-100. ${ }^{1,6}$

Neurofibroma also originate from Schwann cells and must be considered as a differential during histopathologic examination. Neurofibroma does not have a true capsule; have a dense cellular consistency and increased mitosis. Histologic sections show local invasion areas. ${ }^{7}$ There is no Verocay Body formation. Neurofibromas are usually multicentric, which is an important clinical distinction from schwannomas. Neurofibromas may be associated with an entity called 'von Recklinghausen's disease' (cafe-au-lait spots, neurofibromas, optic glioma, Lisch nodules, skeletal malformations, learning disabilities, bilaterally acoustic neuroma etc.). In this disease malignant transformation is as high as $5-30 \%{ }^{8,9}$

The treatment for Schwannoma is wide surgical excision through an approach that allows adequate exposure. Recurrence is rare after total removal. The surgical approach is determined according to the location and extent of the lesion. In some cases, complete excision can be achieved via endonasal endoscopic surgery. In others, depending on the location and extent of the tumor, surgery can involve various combinations of lateral rhinotomy, Caldwell-Luc and external frontoethmoidectomy approaches. ${ }^{10}$

In our literature review, we found only three reported case series on paranasal schwannomas. Hasegawa et al., ${ }^{11}$ reported a series of five cases of sinonasal schwannoma. There were no local recurrences, but the median follow up was only 27 months. Buob et al., ${ }^{12}$ reported five cases, but no distinction was made between the classical form and the cellular variant. These authors had a median follow up of six years, without any local recurrences. Sheikh et al., ${ }^{1}$ followedup for longer period (median 8.1years) with no local recurrence. Therefore local excision without any adjuvant treatment is sufficient. Schwannomas were formerly considered to be radioresistant. Using modern techniques such as intensity modulated radiotherapy (IMRT), the tumour was very well controlled with no recurrence and complications. ${ }^{1}$ Radiotherapy should be reserved for patients who 
are deemed unsuitable for surgery and for patients with malignant schwannomas. $^{6}$

\section{Conclusion}

Schwannoma of the maxillary sinus is a rare pathological entity. It is a benign tumour with potential to local destruction and often the nerve of origin is difficult to establish. Biopsy is required for a definite diagnosis. Complete surgical excision is essential which can achieved by combining endoscopic and open approaches. Every effort should be made to diagnose this condition pre-operatively so that patient can be counselled accordingly and appropriate surgical intervention can be planned. As Schwannomas are not radiosensitive, radiotherapy is reserved for patients deemed unsuitable for surgery and for patients with malignant schwannomas.

\section{Acknowledgments}

None.

\section{Conflicts of interest}

The authors declare that there are no conflicts of interest.

\section{References}

1. Sheikh HY, Chakravarthy RP, Slevin NJ, et al. Benign schwannoma in paranasal sinuses: a clinico-pathological study of five cases, emphasising diagnosing difficulties. J Laryngol Otol. 2008;122(6):598-602.
2. Younis RT, Gross CW, Lazar RH. Schwannomas of the paranasal sinuses. Arch Otolaryngol Head Neck Surg. 1991;117(6):677-680.

3. Shugar MA, Montgomery WW, Reardon EJ. Management of paranasal sinus schwannomas. Ann Otol Rhinol Laryngol. 1982;91(1 Pt 1):65-69.

4. Donnelly MJ, Al-Sader MH, Blaney AW. View from beneath: Pathology in focus. Benign nasal schwannoma. $J$ Laryngol Otol. 1992;106(11):1011-1015.

5. Sanjay K, Gupta SC, Singh PA. Schwannoma of maxillary sinus. Indian J Otolaryngol Head Neck Surg. 2003;55(2):32-135.

6. Colreavy MP, Lacy PD, Hughes J, et al. Head and neck schwannomas - a 10year review. J Laryngol Otol. 2000;114(2):119-124.

7. Chiang CW, Chang YL, Lou PJ. Multicentricity of intraparotid facial nerve schwannomas. Ann Otol Rhinol Laryngol. 2001;110(9): 871-874.

8. Prasad S, Myers N, Kamerer D, et al. Schwannoma of the facial nerve presenting as a parotid mass. Otolarygol Head Neck Surg. 1993;108:76-79.

9. Segas VJ, Kontrogiannis AD, Nomikus PN, et al. A neurilemmoma of the parotid gland: Report of a case. Ear Nose Throat J. 2001;80(7):468-470. .

10. Cakmak O, Yavuz H, Yucel T. Nasal and paranasal sinus schwannomas. Eur Arch Otorhinolaryngol. 2003;260(4):195-197.

11. Hasegawa SL, Mentzel T, Fletcher CD. Schwannomas of the sinonasal tract and nasopharynx. Mod Pathol. 1997;10(8):777-784.

12. Buob D, Wacrenier A, Chevalier D, et al. Schwannoma of the sinonasal tract: a clinicopathologic and immunohistochemical study of 5 cases. Arch Pathol Lab Med. 2003;127(9):1196-1199. 